\title{
Maladie de Huntington et greffes intrastriatales de neurones embryonnaires chez le primate infra-humain
}

La maladie de Huntington est une affection héréditaire à transmission autosomique dominante débutant généralement au milieu de la vie. Elle se caractérise par l'apparition progressive de mouvements choréiques involontaires, de modifications psychiques et d'une démence. Sur le plan neuropathologique, elle est décrite comme une succession de morts neuronales prématurées, intéressant principalement des groupes de neurones situés dans le striatum (noyau caudé et putamen), une structure cérébrale des ganglions de la base, impliquée dans le contrôle du mouvement [1]. Au début, la perte neuronale intéresse essentiellement le noyau caudé et le putamen mais, au fur et à mesure de la progression de la maladie, elle s'étend au pallidum, au cortex cérébral et au thalamus (voir $m / s n^{\circ} 6$, vol. 8, p. 601).

Il est possible de modéliser cette maladie chez le singe par une approche lésionnelle, en administrant localement, dans le striatum, un agoniste du glutamate à des concentrations excitotoxiques. On connaît maintenant avec une bonne précision les conséquences anatomiques, biochimiques, cognitives et comportementales de ce type de lésion chez le rongeur [2, 3]. Les premiers travaux réalisés chez le babouin ont permis d'établir qu'une lésion excitotoxique striatale se traduit (après administration d'un agoniste dopaminergique) par l'apparition d'un syndrome hyperkinétique caractérisé par des dyskinésies oro-faciales, des dyskinésies des extrémités, des dystonies et des mouvements choréiques [4-6]. Ce modèle reproduit donc mieux, cliniquement, la maladie que celui réalisé chez le rongeur, chez lequel la même lésion striatale n'induit pas de mouvements anormaux. Sur le plan neuro-anatomique, la lésion excitotoxique du striatum (noyau caudé et putamen) produit chez le singe (comme chez le rongeur) une dégénérescence des neurones, principa-
Cette dégénérescence des neurones GABAergiques striataux se traduit par une dénervation massive du pallidum et de la substance noire que l'on rend responsable des symptômes moteurs.

Depuis quelques années, des expériences de greffe intrastriatale de tissu embryonnaire ont été réalisées chez le rongeur en utilisant ce modèle excitotoxique de la maladie de Huntington [7-9]. Les résultats montrent que les tissus embryonnaires peuvent survivre et se développer au sein du striatum lésé. En 6-10 semaines, les greffes striatales apparaissent comme un tissu bien délimité, constitué de cellules GABAergiques, cholinergiques et à enképhaline, assez comparables aux cellules striatales adultes. Les études anatomiques faisant appel aux techniques de marquage orthograde et rétrograde montrent que les greffes peuvent recevoir des afférences en provenance du cerveau de l'hôte et, elles-mêmes, innerver des structures cérébrales du cerveau de l'hôte. Ainsi, certains travaux font état d'une restauration du contrôle GABAergique striatal sur le globus pallidus. Néanmoins, si l'on a une bonne connaissance des compensations neurochimiques ou métaboliques produites par ce type de greffes chez le rat, le fait que cet animal ne présente pas de syndrome hyperkinétique après lésion striatale ne permet pas de savoir si les transplants peuvent avoir un effet compensateur sur l'ensemble des symptômes de la maladie. Le travail que nous venons de publier [10] montre que des greffes intrastriatales de tissu striatal embryonnaire ont des effets compensateurs chez le singe dans le modèle excitotoxique de maladie de Huntington.

Une lésion excitotoxique unilatérale du noyau caudé et du putamen a été pratiquée chez 15 babouins Papio papio par administration stéréotaxique intrastriatale d'acide iboténique. Six semaines plus tard, cinq de ces animaux ont été implantés au niveau du striatum lésé, à l'aide d'une suspension de cellules de striatum fotal de rat (âge embryonnaire 14-16 jours). Afin de tester la spécificité tissulaire du tissu greffé, un sixième animal a été implanté avec du tissu embryonnaire provenant du cerveau postérieur de fœtus de rat (âge embryonnaire 14-16 jours). Un septième animal, traité avec le même immunosuppresseur que les animaux greffés (ciclosporine, $10 \mathrm{mg} / \mathrm{kg}$ IM), nous a permis de vérifier que ce traitement ne peut à lui seul modifier les caractéristiques comportementales de la lésion. Tous les animaux (lésés et lésés-greffés) ont été suivis sur le plan comportemental pendant une durée correspondant à neuf semaines après transplantation chez les animaux greffés. L'analyse comportementale est réalisée par enregistrement vidéo et analyse quantitative a posteriori. Une échelle comportementale, validée au cours d'études précédentes $[4,5]$, permet de quantifier l'importance du déficit moteur. Pour tenter d'apporter des informations sur les mécanismes impliqués dans l'effet compensateur des greffes, le traitement immunosuppresseur a été interrompu chez deux animaux, neuf semaines après transplantation de striatum embryonnaire. A ce moment, ces deux animaux présentaient une réduction très significative de leurs symptômes moteurs ( $>50$ \% par rapport aux valeurs après lésion).

Tous les animaux greffés avec du tissu striatal embryonnaire ont montré une diminution progressive des symptômes choréiques (figure 1). L'effet bénéfique des greffes est devenu significatif sept semaines après transplantation et s'est maintenu jusqu'au sacrifice des animaux ou jusqu'à ce que le traitement immunosuppresseur soit interrompu. Aucun effet des greffes de cerveau fœtal postérieur sur l'incidence des symptômes choréiques n'a été noté. La greffe permet de réduire de façon très significative le nombre des dyskinésies 


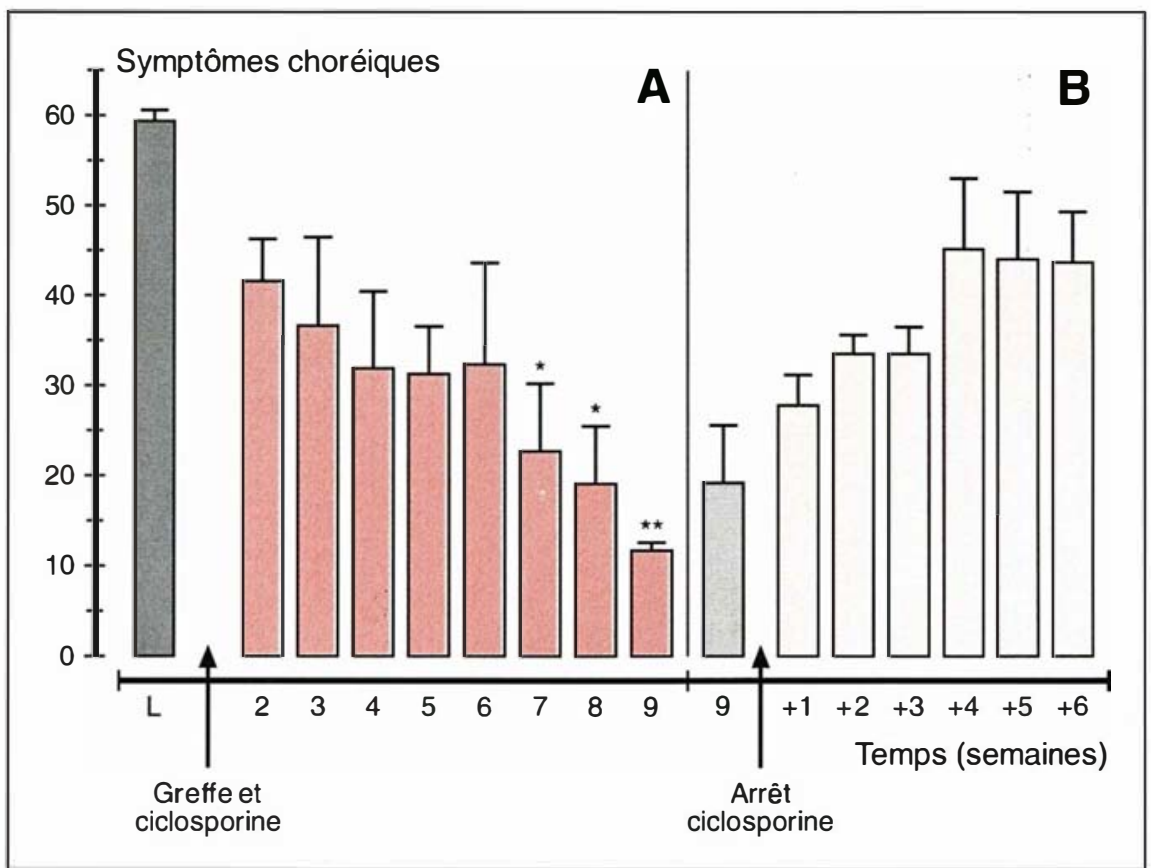

Figure 1. A. Évolution temporelle des scores comportementaux chez les babouins transplantés avec du tissu striatal embryonnaire de rat. La valeur du score comportemental obtenu six semaines après lésion striatale (L) est donnée pour faciliter les comparaisons avec les scores obtenus au cours des neuf semaines qui suivirent l'implantation des cellules fotales. La flèche indique le moment de la transplantation et de l'instauration du traitement immunosuppresseur (régression linéraire et ANOVA : $\left.{ }^{*}=p<0,05,{ }^{* *}=p<0,01\right)$. B. Au cours de la neuvième semaine post-transplantation, le traitement immunosuppresseur a été suspendu (flèche) chez deux babouins transplantés afin de provoquer un rejet immunologique du transplant.

oro-faciales, des dyskinésies des extrémités, des mouvements choréiques et des dystonies. Sur le plan anatomique, les études immunocytochimiques réalisées en utilisant un anticorps spécifique dirigé contre les cellules de rat ont mis en évidence un transplant viable chez tous les animaux transplantés. Pour les deux animaux transplantés chez lesquels le traitement immunosuppresseur a été suspendu, de nombreux signes de rejet du tissu greffé ont, en revanche, été observés.

Cette étude montre que la transplantation intrastriatale de tissu striatal embryonnaire chez le babouin peut améliorer de façon très significative un syndrome hyperkinétique dans un modèle expérimental de maladie de Huntington. Le nombre des symptômes moteurs est réduit de plus de $50 \%$ neuf semaines après la greffe. L'effet des greffes sur la fréquence d'apparition des mouvements anor$\mathrm{m} / \mathrm{s} n^{\circ} 8$, vol. 8 , actobre 92

\section{RÉFÉRENCES}

1. Chesselet MF. La chorée de Huntington. médecine/sciences $1988 ; 4$ : 492-9.

2. Divac I, Marcowitsch HJJ, Pritzel M. Behavioral and anatomical consequences of small intrastriatal injections of kainic acid in the rat. Brain Res $1978 ; 151: 523-32$

3. Sanberg P, Coyle JT. Scientific approaches to Huntington's disease. CRC Crit Rev Clin Neurobiol 1984; 1 : 1-44

4. Hantraye $P$, Riche $D$, Mazière $M$, Mazière B, Loc'h C, Isacson O. Anatomical, behavioural and positron emission tomography studies of unilateral excitotoxic lesions of the baboon caudate-putamen as a primate model of Huntington's disease. In : Crossman AR, Sambrook MA, eds. Neural Mechanisms in Disorders of Movement. Londres : John Libbey and Co, 1989 : 183-93.

5. Hantraye $P$, Riche $D$, Mazière $M$, Isacson $O$. A primate model of Huntington's disease : behavorial and anatomical studies of unilateral excitotoxic lesions of the caudateputamen in the baboon. Exp Neurol 1990 ; 108 : 91-105.

6. Isacson $\mathrm{O}$, Riche $\mathrm{D}$, Hantraye $\mathrm{P}$, Sofroniew MV, Mazière M. A primate model of Huntington's disease : cross species implantations of striatal precursor cells to the excitotoxically lesioned baboon caudate-putamen. Exp Brain Res 1989 ; 75 : 213-20

7. Deckel AW, Robinson RG, Coyle JT, Sanberg PR. Reversal of longterm locomotor abnormalities in the kainic acid model of Huntington's disease by day 18 fetal striatal implants. Eur J Pharmacol 1983 ; 93 : 287-8.

8. Isacson $\mathrm{O}$, Dunnett $\mathrm{SB}$, Bjorklund $\mathrm{A}$. Behavioural recovery in an animal model of Huntington's disease. Proc Natl Acad Sci USA $1986 ; 83$ : 2728-32.

9. Graybiel AM, Liu FC, Dunnett SB Intrastriatal grafts derived from fetal striatal primordia. I. Phenotopy and modular organization. J Neurosci 1989 ; 9 : 3250-71.

10. Hantraye $P$, Riche $D$, Mazière $M$, Isac son $O$. Intrastriatal grafting of fetal striatal cross-species cells ameliorates abnormal movements in a primate model of Huntington's disease. Proc Natl Acad Sci USA 1992 ; 89 : 4187-91.

\section{TIRÉS A PART}

P. Hantraye. 